\title{
Deep Learning Models For Multiword Expression Identification
}

\author{
Waseem Gharbieh and Virendra C. Bhavsar and Paul Cook \\ Faculty of Computer Science, University of New Brunswick \\ Fredericton, NB E3B 5A3 Canada \\ $\{$ waseem.gharbieh, bhavsar, paul.cook\}@unb.ca
}

\begin{abstract}
Multiword expressions (MWEs) are lexical items that can be decomposed into multiple component words, but have properties that are unpredictable with respect to their component words. In this paper we propose the first deep learning models for token-level identification of MWEs. Specifically, we consider a layered feedforward network, a recurrent neural network, and convolutional neural networks. In experimental results we show that convolutional neural networks are able to outperform the previous state-of-the-art for MWE identification, with a convolutional neural network with three hidden layers giving the best performance.
\end{abstract}

\section{Introduction}

Multiword expressions (MWEs) are lexical items that can be decomposed into multiple component words, but have properties that are idiomatic, i.e., marked or unpredictable, with respect to properties of their component words (Baldwin and Kim, 2010). MWEs include a wide range of phenomena such as noun compounds (e.g., speed limit and monkey business), verb-particle constructions (e.g., clean up and throw out), and verb-noun idiomatic combinations (e.g., hit the roof and blow the whistle), as well as named entities (e.g., Prime Minister Justin Trudeau) and proverbs (e.g., Two wrongs don't make a right). One particular challenge for natural language processing (NLP) is MWE identification - i.e., to identify which tokens in running text correspond to MWEs so that they can be analyzed accordingly. The challenges posed by MWEs have led to them to be referred to as a "pain in the neck" for NLP (Sag et al., 2002); nevertheless, incorporating knowledge of MWEs into NLP applications can lead to improvements in tasks including machine translation (Carpuat and Diab, 2010), information retrieval (Newman et al., 2012), and opinion mining (Berend, 2011).

Recent work on token-level MWE identification has focused on methods that are applicable to the full spectrum of kinds of MWEs (Schneider et al., 2014a), in contrast to earlier work that tended to focus on specific kinds of MWEs (Uchiyama et al., 2005; Fazly et al., 2009; Fothergill and Baldwin, 2012). Deep learning is an emerging class of machine learning models that have recently achieved promising results on a range of NLP tasks such as machine translation (Bahdanau et al., 2015; Sutskever et al., 2014), named entity recognition (Lample et al., 2016), natural language generation (Li et al., 2015), and sentence classification (Kim, 2014). Such models have, however, not yet been applied to broad-coverage MWE identification.

In this paper we propose the first deep learning models for broad-coverage MWE identification. Specifically, we propose and evaluate a layered feedforward network, a recurrent neural network, and two convolutional neural networks. We compare these models against the previous state-of-the-art (Kirilin et al., 2016) and several more-traditional supervised machine learning approaches. We show that the convolutional neural networks outperform the previous state-of-the-art. This finding is particularly remarkable given the relatively small size of the training data available, and demonstrates that deep learning models are able to learn well from small datasets. Moreover, we show that our proposed deep learning models are able to generalize more-effectively than previous approaches, based on comparisons between the models' performances on validation and test data. 


\section{Related Work}

MWE identification is the task of determining, at the token level, which words are parts of MWEs, and which are not. For example, in the sentence The staff leaves a lot to be desired (also used in Figure 1) a lot and leaves _to be desired are MWEs. An important part of MWE identification is to be able to distinguish between MWEs and literal combinations that have the same surface form; e.g., kick the bucket is ambiguous between an idiomatic usage - meaning roughly 'die' which is an MWE, and a literal one which is not. Many earlier studies on MWE identification have focused on this type of ambiguity, and treated the problem as one of word sense disambiguation, where literal and idiomatic usages are considered different word senses (Birke and Sarkar, 2006; Katz and Giesbrecht, 2006; Li et al., 2010). Other work has leveraged linguistic knowledge of properties of MWEs in order to make these distinctions (Uchiyama et al., 2005; Fazly et al., 2009; Fothergill and Baldwin, 2012). Crucially, this work has typically focused on specific kinds of MWEs, and has not considered identification of the full spectrum of MWEs.

More-recent work has considered the identification of a wider range of types of MWEs. Brooke et al. (2014) present an unsupervised learning approach to segment a corpus into multiword units based on their predictability. Schneider et al. (2014a) propose methods for broad-coverage MWE identification, and evaluate them on a sizeable corpus (Schneider et al., 2014b). They proposed a supervised learning approach based on the structured perceptron (Collins, 2002). The system labels tokens using the BIO convention, where $B$ indicates the beginning of an MWE, I indicates the continuation of an MWE, and $O$ indicates that the token is not part of an MWE. The model includes features based on part-of-speech tags, MWE lexicons, and Brown clusters (Brown et al., 1992). Qu et al. (2015) later improved upon that system by using skip-gram embeddings (Mikolov et al., 2013) instead of Brown clusters with a variant of conditional random fields. More recently, Constant and Nivre (2016) incorporate MWE identification along with dependency parsing by forming two representations for a sentence: a tree that represents the syntactic dependencies, and a forest of lexical trees that includes the MWEs identified in the sentence.
The recent SemEval shared task on Detecting Minimal Semantic Units and their Meanings (DiMSUM) focused on MWE identification along with supersense tagging (Schneider et al., 2016). The best performing system for MWE identification for this shared task was that of Kirilin et al. (2016) which took into consideration all of the basic features used by Schneider et al. (2014a) and two novel feature sets. The first one is based on the YAGO ontology (Suchanek et al., 2007), where heuristics were applied to extract potential named entities from the ontology. The second feature set was GloVe (Pennington et al., 2014) word embeddings, with the word vectors scaled by a constant and divided by the standard deviation of each of its dimensions. None of the systems that participated in the DiMSUM shared task considered deep learning approaches.

In this paper we propose the first deep learning approaches to MWE identification. We use the DiMSUM data for training and evaluating our models, and compare against the state-of-the-art method of Kirilin et al. (2016). Here we focus solely on the MWE identification task, leaving supersense tagging for future work.

\section{Neural Network Models}

In this section, we discuss the features extracted for the neural network models, and the model architectures. Schneider et al. (2014b) extracted roughly $320 \mathrm{k}$ sparse features. Because of the large input feature space, the only feasible way to train a model on those features is by using a linear classifier. In contrast to Schneider et al. (2014b) our aim is to create dense input features to allow neural network architectures, as well as other machine learning algorithms, to be trained on them. Specifically, we propose three neural network models: a layered feedforward network (LFN), a recurrent neural network (RNN), and a convolutional neural network $(\mathrm{CNN}){ }^{1}$

\subsection{Layered Feedforward Network}

Although LFNs have been used to solve a wide range of classification and regression problems, they have been shown to be less effective for tasks at which deep learning models excel, such as image classification (Krizhevsky et al., 2012) and

\footnotetext{
${ }^{1}$ In preliminary experiments we also considered a sequence-to-sequence model (Cho et al., 2014), but found it to perform poorly relative to the other models, and so do not discuss it further.
} 
machine translation (Bahdanau et al., 2015). The LFN is therefore proposed as a benchmark for comparing the performance of the other architectures, as well as for developing informative input features. Most feature engineering was carried out while developing this model and then transferred to the other architectures.

The composition of the DiMSUM corpus (Schneider et al., 2016), and the token-level lemma and part-of-speech annotations it provides, influenced our feature extraction. Most of the text in the DiMSUM corpus is social media text. The tokens and lemmas were therefore preprocessed by removing \# characters from tokens and lemmas that contain them, and mapping URLs, numbers, and any token or lemma containing the @ symbol to the special tokens URL, NUMBER, and USER, respectively. After pre-processing, distributed representations of all tokens and lemmas were obtained from a skip-gram (Mikolov et al., 2013) model. Specifically, the gensim (Řehưřrek and Sojka, 2010) implementation of skip-gram was trained on a snapshot of Wikipedia from September 2015 to learn 100 dimensional word embeddings. Any token occurring less than 15 times was discarded, the context window was set to 5, the negative sampling rate was set to 5 , and unknown tokens were represented with a zero vector. The part-of-speech tag for each token was also encoded, in this case as a one-hot vector.

Schneider et al. (2014a) included word shape features, which can be informative for the identification of MWEs, especially named entities. We therefore also include word shape features. These are binary features for each token and lemma that capture whether it includes single or double quotes; consists of all capital letters; starts with a capital letter (but is otherwise lowercase); contains a number; includes a \# or @ character; corresponds to a URL; contains any punctuation; and consists entirely of punctuation characters.

Schneider et al. (2014a) include features based on MWE lexicons that represent which tokens and lemmas are potentially part of an MWE and according to which lexicon. We use a script provided by Schneider et al. (2014a) to include these same features in our representation.

Finally, Salton et al. (2016) showed that embedding the entire sentence in which a target MWE occurs was helpful for distinguishing idiomatic from literal verb-noun idiomatic combinations.
We therefore also include a representation for the entire sentence. Specifically, we separately average the skip-gram embeddings for the tokens and lemmas in the sentence containing the target word. These features were then input into an LFN model with a single hidden layer, which we refer to as LFN1.

\subsection{Recurrent Neural Network}

RNNs are a natural fit for many NLP problems due to their ability to model sequences. Here we apply an RNN to broad coverage MWE identification. The token for the current time step is represented using the same features as LFN1 described above, except we do not include the average of the skipgram representations for tokens and lemmas in the same sentence as the target word because we expect the RNN to be able to learn a representation of the sentence by itself. We use a single layer RNN model, referred to as RNN1.

\subsection{Convolutional Neural Network}

CNNs have been shown to be powerful classifiers (Kim, 2014; Kim et al., 2016), and since MWE identification can be formulated as a classification task, CNNs have the potential to perform well on it. The feature representation for the $\mathrm{CNN}$ was split into feature columns to enable the implementation of the convolution layer. Each feature column contains the same features as those for the RNN at each time step but since the CNN does not learn sequential information, a window of feature columns was given as an input.

Multiple filters can then be applied on these feature columns to extract different local features across different window sizes. After finding the optimal number of filters and their sizes, a maxpooling operation is executed on the values extracted by the feature map to form the hidden layer which will be used to produce the predicted output. For our evaluation, we use $\mathrm{CNN}$ architectures with two and three fully connected hidden layers, which we refer to as CNN2 and CNN3, respectively. We observed that CNNs with 2 and 3 hidden layers performed well on the validation set but adding more layers resulted in overfitting. Similarly, adding more hidden layers to the LFN and RNN also resulted in overfitting. 


\section{Data and Evaluation}

This section presents the statistics and structure of the dataset used for this task, as well as the evaluation methodology.

\subsection{Dataset}

We use the DiMSUM dataset (Schneider et al., 2016) for our experiments, which allows for direct comparison with previous results. Table 1 displays the source corpora from which the dataset was constructed; their domain (i.e., reviews, tweets, or TED talks); the number of sentences, words, MWEs, and gappy (i.e., discontiguous) MWES in each source corpus; and the percentage of tokens belonging to an MWE in each source corpus. The dataset is split into training and testing sets such that the testing data contains a novel text type, i.e., TED talks.

For parameter tuning purposes, we also require validation data. We form a validation set from the training data by splitting the training data to create 5 folds, where every fold contained $20 \%$ validation data, and the remaining $80 \%$ was used for training.

\subsection{Structure}

Every line in the dataset provides 8 pieces of information: the numeric position of the token in its sentence; the token itself; its lemmatized form; its part-of-speech tag; its gold-standard MWE tag; the position of the last token that is part of its MWE; its supersense tag; ${ }^{2}$ and the sentence ID. Six MWE tags are used for MWE identification in this dataset, B which indicates the beginning of an MWE, I which indicates the continuation of an MWE, $O$ which indicates that the token is not part of an MWE, $b$ indicates the beginning of a new MWE inside an MWE, $i$ indicates the continuation of the new MWE inside an MWE, and finally, $o$ indicates that the token that is inside an MWE is not part of the nested MWE. This convention assumes that MWEs can only be nested to a depth of one (i.e., an MWE inside an MWE), and that MWEs must be properly nested.

\subsection{Performance Metric}

We use the link-based F-score evaluation metric from Schneider et al. (2014a), which allows

\footnotetext{
${ }^{2}$ Schneider et al. (2014a) consider MWE identification and super-sense tagging. We focus only on MWE identification in this work and so don't use the super-sense tag information provided in the dataset.
}

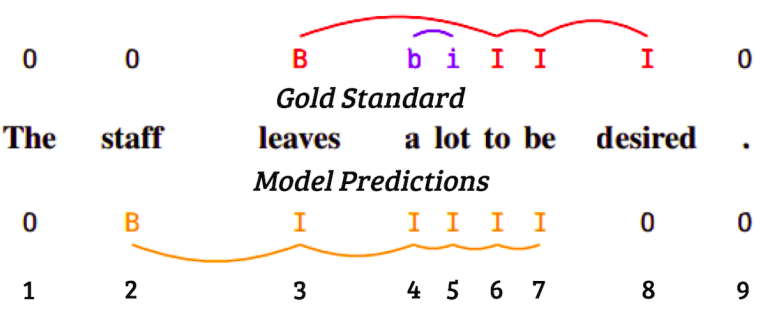

Figure 1: An example of how a model could tag a sequence, along with its gold standard tagging (adapted from Schneider et al. (2016)).

for direct comparison with prior work. Table 1 shows that the percentage of tokens occurring in MWEs ranges from 9-22\%. As such, MWEs occur much less frequently than literal word combinations. This evaluation metric correspondingly puts more emphasis on the ability of the model to detect MWEs rather than literal word combinations.

Figure 1 is a diagram adapted from Schneider et al. (2016) which shows an example of how a model could tag a sequence, as well as its gold standard tagging. The MWE tags on top represent the gold standard, and the MWE tags predicted by a system are shown on the bottom. A link is defined as the path from one token to another, as in Figure 1, regardless of the number of tokens in that path. Precision is calculated as the ratio of the number of correctly predicted links to the total number of links predicted by the model. Recall is calculated in the same way but swapping the gold standard and predicted links.

For example, in Figure 1, the model was able to correctly predict two links. The first link goes from $b$ to $i$ in the gold standard which is matched by a predicted link from token $4-5$ by the model. The second link is from token 6-7 in the gold standard which matches the model's prediction. Since the model predicted five links in total, the precision is $\frac{2}{5}$.

To calculate recall, the roles of the gold standard and model predictions are reversed. This way, three links have been correctly predicted. Two of the three links are the previously mentioned links. The third one is the link from B to I in the gold standard which corresponds to the path from token 3-6. Because there are four links in the gold standard, the recall is therefore $\frac{3}{4}$.

The F-score is then calculated based on precision and recall according to the following equation: 


\begin{tabular}{|c|c|c|c|c|c|c|c|}
\hline Split & Domain & Source corpus & Sentences & Words & MWES & Gappy MWEs & $\%$ tokens in MWE \\
\hline \multirow{4}{*}{ Train } & REVIEWS & STREUSLE 2.1 (Schneider and Smith, 2015) & 3,812 & 55,579 & 3,117 & 397 & $13 \%$ \\
\hline & TWEETS & Lowlands (Johannsen et al., 2014) & 200 & 3,062 & 276 & 5 & $22 \%$ \\
\hline & TWEETS & Ritter (Ritter et al., 2011; Johannsen et al., 2014) & 787 & 15,185 & 839 & 65 & $13 \%$ \\
\hline & & Train Total & 4,799 & 73,826 & 4,232 & 467 & $13 \%$ \\
\hline \multirow{5}{*}{ Test } & REVIEWS & Trustpilot (Hovy et al., 2015) & 340 & 6,357 & 327 & 13 & $12 \%$ \\
\hline & TWEETS & Tweebank (Kong et al., 2014) & 500 & 6,627 & 362 & 20 & $13 \%$ \\
\hline & TED & NAIST-NTT (Cettolo et al., 2012; Neubig et al., 2014) & 100 & 2,187 & 93 & 2 & $9 \%$ \\
\hline & TED & IWSLT test (Cettolo et al., 2012) & 60 & 1,329 & 55 & 1 & $9 \%$ \\
\hline & & Test Total & 1,000 & 16,500 & 837 & 36 & $12 \%$ \\
\hline
\end{tabular}

Table 1: Statistics describing the composition of the DiMSUM dataset.

$$
\frac{1}{F}=\frac{1}{2}\left(\frac{1}{P}+\frac{1}{R}\right)
$$

where $F$ is the F-score, and $P$ and $R$ are precision and recall, respectively.

\section{Parameter Settings}

In this section, the architecture and parameters of all neural network models are presented in detail. The cost function used to train the neural network models was based on the cost function used by Schneider et al. (2014a) for this task:

$$
\operatorname{cost}=\sum_{i=1}^{\left|\bar{y}_{i}\right|} c\left(\bar{y}_{i}, y_{i}\right)
$$

where $\bar{y}_{i}$ is the $i$ th gold standard MWE tag, and $y_{i}$ is the $i$ th MWE tag predicted by the neural network model. To ensure that the MWE tag predicted by the neural network is a probability distribution, the output layer of all neural models was the softmax layer. The function $c$ in Equation 2 is defined as:

$$
c\left(\bar{y}_{i}, y_{i}\right)=\bar{y}_{i} \log \left(y_{i}\right)+\rho\left(\bar{y}_{i} \epsilon\{B\} \wedge y_{i} \epsilon\{O\}\right)
$$

Some MWE tag sequences are invalid, for example, a B followed immediately by an 0 (because MWEs are composed of multiple tokens), and similarly, an 0 cannot occur immediately before an I (because the beginning of every MWE must be tagged with a $B$ ). We therefore use the Viterbi algorithm on the output of the neural network models to obtain the valid MWE tag sequence with the highest probability. In preliminary experiments we observed that setting all valid transitions to be of equal probability, and the probability of all invalid transitions to 0 , performed best, and therefore use this strategy.

\subsection{Layered Feedforward Network}

The LFN was used as a benchmark neural network model against which the performance of the other deep learning models was compared. The parameters that had to be tuned for this model were the size of the context window, the misclassification penalty $\rho$ (in Equation 3), the number of neurons in each hidden layer, the number of iterations before training is stopped, and the dropout rate. Optimizing these parameters is important as they greatly influence the performance of the LFN. For all models considered, all parameter tuning was done using the validation data; the test data was never used for setting parameters.

Context window of sizes of 1,2 , and 3 tokens to the left and right were considered. A larger context window allows the model to see additional tokens, but also makes the training process longer and more prone to overfitting. In the case of $\rho$, we investigated setting it between 40 and 100. A small value of $\rho$ would cause the model to have high precision but low recall, while a larger value would trade off recall for precision. The number of neurons in the hidden layer that was examined ranged from 100 to 1200 . Adding more neurons in a hidden layer, and introducing more hidden layers, allows the LFN to model more complex functions, but can also make it more prone to overfitting. We avoid overfitting by stopping training after a defined number of iterations (by observing the performance of the model on the validation set), and by using dropout (Srivastava et al., 2014). Dropout combats overfitting by randomly switching off a percentage of the neurons in a hidden layer during training, which allows a neural network to be more robust in its predictions as it 
decreases the association between neurons. It also has the same effect as ensembling multiple neural network models because different neurons are switched on and off in every training iteration. The dropout rates that we considered ranged from 0.4 to 0.6 .

After running multiple experiments, the best performing LFN model (LFN1) had a context window of size 1 , which means that the features for the tokens before and after the target token were input into the LFN along with the features of the target token. The value of $\rho$ was set to 50, and the LFN had a single hidden layer containing 1000 neurons with the tanh activation function. The LFN was trained for 1000 iterations with a dropout rate of 0.5 .

\subsection{Recurrent Neural Network}

As previously mentioned in Section 3.2, RNNs are a natural fit to many NLP problems due to their ability to model sequences. At each timestep, the features for a token were input into the RNN which then output the corresponding MWE tag for that token. Many of the parameters that had to be tuned for the LFN had to be tuned for the RNN as well: $\rho$ ranged from 10 to 50 ; the number of neurons in each hidden layer ranged from 50 to 300 ; the dropout rate ranged from 0.5 to 1 ; and we again tuned the number of iterations before training is stopped. ${ }^{3}$ Parameters specific to the RNN model that had to be tuned include whether the RNN is unidirectional or bidirectional, and the cell type, where we consider a fully connected RNN, an LSTM cell, and a GRU cell.

After observing the performance of the RNN on the validation set, the best performing RNN model (RNN1) was a bidirectional LSTM with $\rho$ set to 25 , with a single hidden layer containing 100 neurons. It was trained for 60 iterations with no dropout. This indicates that the LSTM cell was able to handle the complexity of the sequences of tokens without requiring regularization.

As we will see in Section 6, RNN1 unfortunately did not perform as well as the other neural network models. We therefore attempted to improve its performance using two additional approaches. In the first approach, the RNN LSTM was orthogonally initialized. Saxe et al. (2014) showed that orthogonally initializing RNNs led to

\footnotetext{
${ }^{3}$ We choose parameter settings to explore based on performance on the validation data, and so consider different parameter settings here than for LFN1.
}

better learning in deep neural networks. Nevertheless, orthogonal initialization did not seem to have an effect on the performance of RNN1. In the second approach, the dataset was artificially expanded by splitting the input sentences on punctuation. This provided more "sentences" for the RNN LSTM to learn from, but again did not improve performance.

\subsection{Convolutional Neural Network}

Every token was represented by a feature column and these feature columns were then concatenated to form the input to the CNN. A convolutional layer was then applied to the input and then maxpooled to form the hidden layer which was used to produce the predicted output. There were again many parameters to optimize in the CNN. We considered the same settings for the context window size as for LFN1, i.e., 1, 2, and 3 tokens to the left and right. The number of neurons in each hidden layer ranged from 25 to 200 . In contrast to LFN1 and RNN1, here we consider varying numbers of fully connected hidden layers from 1-3. The dropout rate at the fully connected layers, as well as the convolutional layer, ranged from 0.3 to 1 , and $\rho$ ranged from 10 to 30. Parameters specific to the convolutional neural network that were optimized were the number of filters, which ranged from 100 to 500, and spanned 1, 2, or 3 feature columns, and the types of convolution and pooling operations that were performed. Having a large number of filters can cause the network to pick up noise patterns which makes the $\mathrm{CNN}$ overfit. The size of the filters and the types of convolution and pooling operations is largely dependent on the data and were optimized according to the performance of the model on the validation set.

We experiment with two CNN models, the best performing $\mathrm{CNN}$ model with two hidden layers (CNN2) and the best performing $\mathrm{CNN}$ model with three hidden layers (CNN3). CNN2 was trained for 600 iterations and had a context window of size 1 and $\rho$ equal to 20, with 250 filters that spanned 2 feature columns, and 200 filters that spanned all 3 feature columns. Narrow convolution was used which produced a hidden layer with 450 neurons. This layer was then input into another hidden layer containing 50 neurons with the sigmoid activation function before being passed to the output softmax layer. 
CNN3 is similar to CNN2 but was trained for 900 iterations and had the 450 neuron hidden layer feed to a hidden layer containing 100 neurons with the sigmoid activation function. The output of that layer was then passed to another layer containing 50 neurons with the tanh activation function before being passed to the output softmax layer. The intuition behind the tanh activation function for the last hidden layer is that the layer before it has the sigmoid activation function. This means that the values that are passed to the last hidden layer are between 0 and 1 multiplied by the weights between the two layers. Since these weights can be negative, a sigmoid function that can deal with negative values is required, and the tanh function satisfies this requirement. Both models have a dropout rate of $60 \%$ on the convolutional and hidden layers. They were also given batches of 6000 random examples at each training iteration.

\subsection{Traditional Machine Learning Models}

To demonstrate the effectiveness of neural network models, we compare them against moretraditional, non-neural machine learning models. Here we consider $k$-nearest neighbour, random forests, logistic regression, and gradient boosting. ${ }^{4}$ These models were given the same features that were input into LFN1, and parameter tuning was also carried out on the validation set. For the $k$ nearest neighbour algorithm, $k$ was set to 3 , and the points were weighted by the inverse of their distance. For random forests, 100 estimators were used while multiplying the penalty of misclassifying any class other than 0 as 0 by 1.2. In the case of logistic regression, L2 regularization was utilized with a regularization factor of 0.5 . For gradient boosting, 100 estimators with a maximum depth of 13 nodes were used. Using a larger number of estimators for random forest and gradient boosting has shown to improve their cross validation performance. However, the point of diminishing returns was found to be at around 50 estimators, and it was clear that increasing the number of estimators above 100 would not yield any significant increase in performance. Added to that, with gradient boosting, the cross validation performance also increased with the maximum node depth, but the point of diminishing returns was found to be at around 9, and it was clear that increasing the

\footnotetext{
${ }^{4}$ In preliminary experiments we also considered an SVM, but found the training time to be impractical, and so did not consider it further.
}

maximum depth beyond 13 would not yield any significant increase in performance.

\subsection{Implementation Details}

Overall, 983 features were input into the LFN and traditional machine learning models, and more than 50 parameter combinations were examined. Every LFN model required up to 2 days of training. For the RNN, every token was represented by a feature vector of length 257 , and took around 10 hours to train. More than 30 parameter combinations were examined for the RNN model. Every feature column in the CNN model contained 257 features, this amounts to a total of 771 input features. More than 130 parameter combinations were tested for the CNN, and it required around 12 hours of training. Tensorflow (et al., 2015) version 0.12 was used to implement the neural network models, and scikit-learn (Pedregosa et al., 2011) was used to implement the traditional machine learning models. The experiments were run on 2 GHz Intel Xeon E7-4809 v3 CPUs.

\section{Results}

The average F-score of the models on the five fold cross validation set, and their F-score on the test set, along with their generalization, is shown in Table 2. All models except for that of Kirilin et al. (2016) - which was already optimized for this task by its authors - were run on the validation set to tune their parameters. To evaluate the performance of the models on the test set, the models were trained on the entire training set (which includes the validation splits) and then tested on the test set.

We first consider the traditional machine learning models. Amongst these models, gradient boosting performed best on the validation set, which can be attributed to the ability of gradient boosting to learn complex functions and its robustness to outliers. However, it did not perform as well on the test set, where logistic regression performed best, and achieved the best generalization out of the traditional machine learning models. This shows that relatively many instances in the test set can be correctly classified by using a hyperplane to separate the dense feature representations.

Turning to the proposed neural network models, LFN1 is indeed a strong baseline for this task. This model achieved an F-score on the test set that 


\begin{tabular}{ccccc}
\hline \multirow{2}{*}{ Model Class } & \multirow{2}{*}{ Model } & \multicolumn{2}{c}{ F-score } & \multirow{2}{*}{ Validation Set } \\
& & Test Set & \multirow{2}{*}{ Generalization } \\
\hline \multirow{2}{*}{ Traditional } & k-Nearest Neighbour & 48.35 & 31.30 & $64.74 \%$ \\
Machine & Random Forest & 52.26 & 32.02 & $61.27 \%$ \\
Learning Models & Logistic Regression & 57.68 & 53.37 & $92.53 \%$ \\
& Gradient Boosting & 64.98 & 48.79 & $75.08 \%$ \\
\hline \multirow{2}{*}{ Neural Network } & LFN1 & 66.48 & 57.99 & $87.23 \%$ \\
Models & RNN1 & 56.96 & 53.07 & $\mathbf{9 3 . 1 7 \%}$ \\
& CNN2 & 66.95 & 59.18 & $88.39 \%$ \\
\multirow{2}{*}{ Baseline Models } & CNN3 & 67.40 & $\mathbf{5 9 . 9 6}$ & $88.96 \%$ \\
& Schneider and Smith (2015) & $\mathbf{6 7 . 8 4}$ & 57.74 & $85.11 \%$ \\
\hline
\end{tabular}

Table 2: The average F-score of each model on the 5 fold cross validation set, and their F-score on the test set, along with their generalization. The best performance in each column is shown in boldface.

comes close to the previous state-of-the-art of Kirilin et al. (2016). RNN1 achieved the best generalization out of all models considered; however, it performed relatively poorly compared to the other neural network models on both the validation and test sets. The CNN models, CNN2 and CNN3, both improved over the previous best results on the test set — with CNN3 achieving the best F-score overall - and outperformed all other models except for (Schneider et al., 2014a) on the validation set. This shows that the CNN filters were able to learn what makes a feature column a part of an MWE or not. That CNN3 outperforms CNN2 further shows that adding an extra hidden layer for the $\mathrm{CNN}$ model improves its performance as it is able to handle more complex mappings. Moreover, the training data for this task is relatively small; it consists of less than 5,000 sentences. These results therefore further show that convolutional neural networks can still achieve good performance when the amount of training data available is limited.

The highest F-score on the test set - achieved by CNN3 - is 59.96. This shows that the task is quite difficult, and suggests that there is scope for further improvements. One issue, however, is that there are notable inconsistencies in the annotations in the dataset. For example, the expression a few is labeled as an MWE 15 out of 32 times in the training set, even though there appears to be no variation in its usage. Recent efforts have, however, proposed semi-automated methods for resolving these inconsistencies (Chan et al., 2017).

\section{Conclusions and Future Work}

We proposed and evaluated the first neural network approaches for multiword expression identification, and compared their performance against the previous state-of-art, and more-traditional machine learning approaches. We showed that our proposed approach based on a convolutional neural network (CNN2 and CNN3) outperformed the previous state-of-the-art for this task. Therefore, although the task is inherently sequential, formulating it as a classification task enabled the CNN models to perform well on it. This finding suggests that deep learning methods can still be effective when only limited amounts of training data are available. Furthermore, the proposed neural network-based approaches were able to generalize more-effectively than previous approaches.

In future work, we intend to carry out an indepth analysis of the errors committed by the neural network models. Additionally, an ablation study of the features can be conducted to determine the effect of each feature set on the overall performance of the models. The proposed deep learning models can also be extended to predict supersense tags in addition to the MWE tags. In particular, we intend to compare the performance of a single model that predicts the supersense and MWE tags, versus two separate models for each task. Furthermore, we plan to measure the impact of MWE identification on downstream NLP tasks by incorporating the predicted MWE tags into applications such as machine translation. 


\section{Acknowledgments}

This work is financially supported by the Natural Sciences and Engineering Research Council of Canada, the New Brunswick Innovation Foundation, and the University of New Brunswick.

\section{References}

Dzmitry Bahdanau, Kyunghyun Cho, and Yoshua Bengio. 2015. Neural machine translation by jointly learning to align and translate. In International Conference on Learning Representations (ICLR2015).

Timothy Baldwin and Su Nam Kim. 2010. Handbook of natural language processing. In Nitin Indurkhya and Fred J. Damerau, editors, Handbook of Natural Language Processing, CRC Press, Boca Raton, USA. 2nd edition.

Gábor Berend. 2011. Opinion expression mining by exploiting keyphrase extraction. In Proceedings of 5th International Joint Conference on Natural Language Processing. Chiang Mai, Thailand, pages 1162-1170.

Julia Birke and Anoop Sarkar. 2006. A clustering approach for nearly unsupervised recognition of nonliteral language. In Proceedings of the 11th Conference of the European Chapter of the Association for Computational Linguistics (EACL-2006). Trento, Italy, pages 329-336.

Julian Brooke, Vivian Tsang, Graeme Hirst, and Fraser Shein. 2014. Unsupervised multiword segmentation of large corpora using prediction-driven decomposition of n-grams. In COLING 2014, 25th International Conference on Computational Linguistics, Proceedings of the Conference: Technical Papers. Dublin, Ireland, pages 753-761.

Peter F. Brown, Vincent J. Della Pietra, Peter V. de Souza, Jennifer C. Lai, and Robert L. Mercer. 1992. Class-based n-gram models of natural language. Computational Linguistics 18(4):467-479.

Marine Carpuat and Mona Diab. 2010. Task-based evaluation of multiword expressions: a pilot study in statistical machine translation. In Human Language Technologies: The 2010 Annual Conference of the North American Chapter of the Association for Computational Linguistics. Los Angeles, California, pages 242-245.

Mauro Cettolo, Christian Girardi, and Marcello Federico. 2012. Web inventory of transcribed and translated talks. In Proceedings of the 16th Annual Conference of the European Association for Machine Translation (EAMT 2012). Trento, Italy, pages 261268.

King Chan, Julian Brooke, and Timothy Baldwin. 2017. Semi-automated resolution of inconsistency for a harmonized multiword expression and dependency parse annotation. In Proceedings of the 13th Workshop on Multiword Expressions (MWE 2017). Valencia, Spain, pages 187-193.

Kyunghyun Cho, Bart van Merrienboer, Çaglar Gülçehre, Dzmitry Bahdanau, Fethi Bougares, Holger Schwenk, and Yoshua Bengio. 2014. Learning phrase representations using RNN encoder-decoder for statistical machine translation. In Proceedings of the 2014 Conference on Empirical Methods in Natural Language Processing, A meeting of SIGDAT, a Special Interest Group of the ACL. Doha, Qatar, pages 1724-1734.

Michael Collins. 2002. Discriminative training methods for hidden markov models: Theory and experiments with perceptron algorithms. In Proceedings of the 2002 Conference on Empirical Methods in Natural Language Processing (EMNLP 2002). Philadelphia, USA, pages 1-8.

Matthieu Constant and Joakim Nivre. 2016. A transition-based system for joint lexical and syntactic analysis. In Proceedings of the 54th Annual Meeting of the Association for Computational Linguistics. Berlin, Germany, pages 161-171.

Martín Abadi et al. 2015. TensorFlow: Largescale machine learning on heterogeneous systems. Software available from tensorflow.org. http://tensorflow.org/.

Afsaneh Fazly, Paul Cook, and Suzanne Stevenson. 2009. Unsupervised type and token identification of idiomatic expressions. Computational Linguistics 35(1):61-103.

Richard Fothergill and Timothy Baldwin. 2012. Combining resources for mwe-token classification. In *SEM 2012: The First Joint Conference on Lexical and Computational Semantics - Volume 1: Proceedings of the main conference and the shared task, and Volume 2: Proceedings of the Sixth International Workshop on Semantic Evaluation (SemEval 2012). Montréal, Canada, pages 100-104.

Dirk Hovy, Anders Johannsen, and Anders Søgaard. 2015. User review sites as a resource for large-scale sociolinguistic studies. In Proceedings of the 24th International Conference on World Wide Web. Florence, Italy, pages $452-461$.

Anders Johannsen, Dirk Hovy, Héctor Martínez Alonso, Barbara Plank, and Anders Søgaard. 2014. More or less supervised supersense tagging of twitter. In Proceedings of the Third Joint Conference on Lexical and Computational Semantics (*SEM 2014). Dublin, Ireland, pages $1-11$.

Graham Katz and Eugenie Giesbrecht. 2006. Automatic identification of non-compositional multiword expressions using latent semantic analysis. In 
Proceedings of the Workshop on Multiword Expressions: Identifying and Exploiting Underlying Properties. Sydney, Australia, pages 12-19.

Yoon Kim. 2014. Convolutional neural networks for sentence classification. In Proceedings of the 2014 Conference on Empirical Methods in Natural Language Processing, EMNLP, A meeting of SIGDAT, a Special Interest Group of the ACL. Doha, Qatar, pages 1746-1751.

Yoon Kim, Yacine Jernite, David Sontag, and Alexander M. Rush. 2016. Character-aware neural language models. In Proceedings of the Thirtieth AAAI Conference on Artificial Intelligence. Phoenix, Arizona, USA, pages 2741-2749.

Angelika Kirilin, Felix Krauss, and Yannick Versley. 2016. ICL-HD at semeval-2016 task 10: Improving the detection of minimal semantic units and their meanings with an ontology and word embeddings. In Proceedings of the 10th International Workshop on Semantic Evaluation, SemEval@NAACL-HLT. San Diego, CA, USA, pages 937-945.

Lingpeng Kong, Nathan Schneider, Swabha Swayamdipta, Archna Bhatia, Chris Dyer, and Noah A. Smith. 2014. A dependency parser for tweets. In Proceedings of the 2014 Conference on Empirical Methods in Natural Language Processing, A meeting of SIGDAT, a Special Interest Group of the ACL. Doha, Qatar, pages 1001-1012.

Alex Krizhevsky, Ilya Sutskever, and Geoffrey E Hinton. 2012. ImageNet classification with deep convolutional neural networks. In F. Pereira, C. J. C. Burges, L. Bottou, and K. Q. Weinberger, editors, Advances in Neural Information Processing Systems 25, Curran Associates, Inc., pages 1097-1105.

Guillaume Lample, Miguel Ballesteros, Sandeep Subramanian, Kazuya Kawakami, and Chris Dyer. 2016. Neural architectures for named entity recognition. In NAACL HLT 2016, The 2016 Conference of the North American Chapter of the Association for Computational Linguistics: Human Language Technologies. San Diego, California, USA, pages 260 270.

Jiwei Li, Minh-Thang Luong, and Dan Jurafsky. 2015. A hierarchical neural autoencoder for paragraphs and documents. In Proceedings of the 53rd Annual Meeting of the Association for Computational Linguistics and the 7th International Joint Conference on Natural Language Processing of the Asian Federation of Natural Language Processing, ACL, Volume 1: Long Papers. Beijing, China, pages 1106-1115.

Linlin Li, Benjamin Roth, and Caroline Sporleder. 2010. Topic models for word sense disambiguation and token-based idiom detection. In Proceedings of the 48th Annual Meeting of the Association for Computational Linguistics. Uppsala, Sweden, pages 1138-1147.
Tomas Mikolov, Ilya Sutskever, Kai Chen, Greg S Corrado, and Jeff Dean. 2013. Distributed representations of words and phrases and their compositionality. In C. J. C. Burges, L. Bottou, M. Welling, Z. Ghahramani, and K. Q. Weinberger, editors, $A d$ vances in Neural Information Processing Systems 26, Curran Associates, Inc., pages 3111-3119.

Graham Neubig, Katsuhito Sudoh, Yusuke Oda, Kevin Duh, Hajime Tsukada, and Masaaki Nagata. 2014. The NAIST-NTT TED talk treebank. In International Workshop on Spoken Language Translation (IWSLT). Lake Tahoe, USA.

David Newman, Nagendra Koilada, Jey Han Lau, and Timothy Baldwin. 2012. Bayesian text segmentation for index term identification and keyphrase extraction. In Proceedings of COLING 2012. Mumbai, India, pages 2077-2092.

F. Pedregosa, G. Varoquaux, A. Gramfort, V. Michel, B. Thirion, O. Grisel, M. Blondel, P. Prettenhofer, R. Weiss, V. Dubourg, J. Vanderplas, A. Passos, D. Cournapeau, M. Brucher, M. Perrot, and E. Duchesnay. 2011. Scikit-learn: Machine learning in Python. Journal of Machine Learning Research 12:2825-2830.

Jeffrey Pennington, Richard Socher, and Christopher Manning. 2014. Glove: Global vectors for word representation. In Proceedings of the 2014 Conference on Empirical Methods in Natural Language Processing (EMNLP). Doha, Qatar, pages 15321543.

Lizhen Qu, Gabriela Ferraro, Liyuan Zhou, Weiwei Hou, Nathan Schneider, and Timothy Baldwin. 2015. Big data small data, in domain out-of domain, known word unknown word: The impact of word representations on sequence labelling tasks. In Proceedings of the Nineteenth Conference on Computational Natural Language Learning. Beijing, China, pages 83-93.

Radim Řehůřek and Petr Sojka. 2010. Software Framework for Topic Modelling with Large Corpora. In Proceedings of the LREC 2010 Workshop on New Challenges for NLP Frameworks. Valletta, Malta, pages $45-50$.

Alan Ritter, Sam Clark, Mausam, and Oren Etzioni. 2011. Named entity recognition in tweets: An experimental study. In Proceedings of the 2011 Conference on Empirical Methods in Natural Language Processing, A meeting of SIGDAT, a Special Interest Group of the ACL. Edinburgh, UK, pages 15241534.

Ivan A. Sag, Timothy Baldwin, Francis Bond, Ann Copestake, and Dan Flickinger. 2002. Multiword expressions: A pain in the neck for NLP. In Proceedings of the Third International Conference on Intelligent Text Processing and Computational Linguistics (CICLING 2002). pages 1-15. 
Giancarlo Salton, Robert Ross, and John Kelleher. 2016. Idiom token classification using sentential distributed semantics. In Proceedings of the 54th Annual Meeting of the Association for Computational Linguistics (Volume 1: Long Papers). Berlin, Germany, pages 194-204.

Andrew M. Saxe, James L. McClelland, and Surya Ganguli. 2014. Exact solutions to the nonlinear dynamics of learning in deep linear neural networks. In International Conference on Learning Representations (ICLR2014).

Nathan Schneider, Emily Danchik, Chris Dyer, and A. Noah Smith. 2014a. Discriminative lexical semantic segmentation with gaps: Running the mwe gamut. Transactions of the Association for Computational Linguistics (TACL) 2:193-206.

Nathan Schneider, Dirk Hovy, Anders Johannsen, and Marine Carpuat. 2016. SemEval-2016 task 10: Detecting minimal semantic units and their meanings (DiMSUM). In Proceedings of the 10th International Workshop on Semantic Evaluation,SemEval@NAACL-HLT. San Diego, CA, USA, pages 546-559.

Nathan Schneider, Spencer Onuffer, Nora Kazour, Emily Danchik, Michael T. Mordowanec, Henrietta Conrad, and Noah A. Smith. 2014b. Comprehensive annotation of multiword expressions in a social web corpus. In Proceedings of the Ninth International Conference on Language Resources and Evaluation (LREC-2014). Reykjavik, Iceland, pages 455-461.

Nathan Schneider and A. Noah Smith. 2015. A corpus and model integrating multiword expressions and supersenses. In Proceedings of the 2015 Conference of the North American Chapter of the Association for Computational Linguistics: Human Language Technologies. Denver, Colorado, pages 1537-1547.

Nitish Srivastava, Geoffrey E. Hinton, Alex Krizhevsky, Ilya Sutskever, and Ruslan Salakhutdinov. 2014. Dropout: a simple way to prevent neural networks from overfitting. Journal of Machine Learning Research 15(1):1929-1958.

Fabian M. Suchanek, Gjergji Kasneci, and Gerhard Weikum. 2007. Yago: a core of semantic knowledge. In Proceedings of the 16th International Conference on World Wide Web. Banff, Alberta, Canada, pages 697-706.

Ilya Sutskever, Oriol Vinyals, and Quoc V Le. 2014. Sequence to sequence learning with neural networks. In Z. Ghahramani, M. Welling, C. Cortes, N. D. Lawrence, and K. Q. Weinberger, editors, Advances in Neural Information Processing Systems 27, Curran Associates, Inc., pages 3104-3112.

Kiyoko Uchiyama, Timothy Baldwin, and Shun Ishizaki. 2005. Disambiguating Japanese compound verbs. Computer Speech and Language, Special Issue on Multiword Expressions 19(4):497-512. 